STUPP-05-180

TUM-HEP-581/05

\title{
Monoenergetic Neutrino Beam for Long Baseline Experiments
}

\author{
Joe Sato $^{1,2, *}$ \\ ${ }^{1}$ Department of Physics, Saitama University, \\ Shimo-okubo, Sakura-ku, Saitama, 338-8570, Japan \\ ${ }^{2}$ Physik-Department Technische Universität München, \\ James-Franck-Strasse 85748 Garching, Germany
}

\begin{abstract}
In an electron capture process by a nucleus, emitted neutrinos are monoenergetic. By making use of it, we study how to get a completely monoenergetic neutrino beam in a long baseline experiment.
\end{abstract}

PACS numbers: 14.60.Pq,23.40.Bw

Keywords: long baseline experiment, neutrino oscillation, monoenergetic neutrino, $e^{-}$capture, $\beta^{+}$decay

*Electronic address: joe@phy.saitama-u.ac.jp 


\section{INTRODUCTION}

Numerous observations on neutrinos from the sun[1], the atmosphere 2], the reactor [3], and the accelerator [4] suggest that neutrinos are massive and hence there are mixings in the lepton sector.

Within the three generations, two of the mixing angles and the two mass differences are well determined. [5] To determine these parameters much more precisely and to observe effects from the other two mixing parameters, $\theta_{13}$ and $\mathrm{CP}$ phase $\delta$, there are several ideas proposed for next generation of neutrino oscillation experiments. [6, 7], 8, 9]

For a precision measurement, it is apparently better that we make an experiment using neutrinos with manageable and precisely known energy. In this respect we consider making use of a nucleus which absorbs an electron and emits a neutrino:

$$
(Z, A)+e^{-} \rightarrow(Z-1, A)+\nu_{e}
$$

where $Z$ is the electric charge of the mother nucleus and $A$ is its mass number. In this case neutrinos have a line spectrum and its energy is precisely known. Therefore by accelerating the mother nuclei appropriately with the Lorenz boost factor $\gamma_{m}$, we can control neutrino energy and make use of monoenergetic neutrinos in an oscillation experiment.

The experimental setup is very simple. We need an accumulating ring as usual[8] to circulate nucleus. This ring equips an electron injection at the entrance of the decay section with its length $X$ and an apparatus for separation of nuclei and electrons at the exit of the decay section. The injected electrons must be tuned precisely so that their boost factor $\gamma_{e}$ must be same as that of nuclei $\gamma_{m}, \gamma_{e}=\gamma_{m} .{ }^{1}$ The separation section at the exit must be also constructed so that it can separate the nuclei and electron properly to circulate the nuclei till its decay. It may be implemented by photon injection and a strong magnet.

The range of neutrino energy, $E_{\nu}$, in the laboratory system is given by

$$
0<E_{\nu}<2 \gamma_{m} Q
$$

\footnotetext{
${ }^{1}$ When a free electron falls into an orbit of a nucleus, a photon with energy of minding energy, $B$, of O(1-10) $\mathrm{keV}$ is emitted. It gives uncertainty of momentum $B^{2} / M \sim \mathrm{O}(1) \mathrm{eV}$ for a nucleus with mass $M$ in the rest frame of the nucleus. However it is much smaller than the nucleus momentum in the laboratory frame so that we can safely ignore this effect. Also it takes a time of $\mathrm{O}\left(10^{-9} / Z^{4}\right)$ sec for an electron to fall into an orbit of a nucleus which is much shorter than the duration within which the nucleus stays in the decay section in the rest frame, $X / \gamma_{m}>10^{-8}$ sec. Therefore we can assume that it occurs instantly.
} 
where $Q$ is the energy difference between the mother and the daughter nuclei and $Q \ll M$.

The appropriate energy for the experiment is derived from the baseline length $L$ and the relevant mass square difference $\delta m^{2}$ :

$$
\left.\frac{\delta m^{2} L}{4 E_{\nu}}\right|_{E_{\nu}=2 \gamma_{m} Q}=P,
$$

where $P$ is the oscillation phase at the maximum energy of neutrino which is determined from the physics goal. For example, if one wants to observe the oscillation at the first maximum, then $P=\pi / 2$. From eq.(3),

$$
\gamma_{m}=\frac{\delta m^{2} L}{8 P} \frac{1}{Q} .
$$

Since in the rest frame of the mother nuclei, the distance between the decay pipe and the detector is $L^{\prime} \equiv L / \gamma_{m}$, the larger $\gamma_{m}$ means the higher neutrino flux at a detector. It scales proportionally to $\gamma_{m}^{2}$. It means from eq.(44) that the lower $Q$ value is better. However a lower $Q$ means, in general, a larger half-life $\tau$. The mother nuclei should capture an electron frequently enough, otherwise we cannot get neutrino beam of a sufficient strength. It means

$$
\tau \gamma_{m}<T \Rightarrow \frac{\delta m^{2} L}{8 P T}<Q / \tau
$$

where $T$ is an appropriate time interval within which we require that all the mother nuclei should experience the process (11). Therefore, since in this kind of experiments data are taken for several years, $T$ is of order a month or at most a year. This requires that $\gamma_{m}$ should be smaller and it conflicts with the requirement to get a higher-flux neutrino beam mentioned below eq. (44). To satisfy both the requirements, we have to find a nucleus which has a smaller $Q$ value and a shorter half-life $\tau$. In the following $\gamma_{m} \gg 1$ and nuclei mass $M \gg Q$ are used to derive equations.

Here we examine the theoretical aspects of this idea in more detail.

Case (i) Purely monoenergetic neutrino:

As one of the first candidates we study here ${ }_{50}^{110} \mathrm{Sn}$. Theoretically this gives the best example for our scenario. Its half-life $\tau_{\mathrm{Sn}}$ is 4.11 hour. Its $J^{P}$ is $0^{+}$. It decays into the excited state of ${ }_{49}^{110} \mathrm{In}$, with $1^{+}$whose energy level is $343 \mathrm{keV}$. Since the mass difference is $638 \mathrm{keV}$, [10] the energy difference between neutral ${ }_{50}^{110} \mathrm{Sn}$ and ${ }_{49}^{110} \mathrm{In}, \Delta_{\mathrm{Sn}}$, is $295 \mathrm{keV}$, that is, the energy of the emitted neutrino is $295 \mathrm{keV}$ minus to the binding energy. For example, 
since the $K$ shell binding energy, $E_{\mathrm{In}}^{K}$ of ${ }_{49}^{110} \mathrm{In}$ is $28 \mathrm{keV}[11]^{2}$, the emitted neutrino energy in the rest frame of $\mathrm{Sn}, Q_{\mathrm{Sn}}=\Delta_{\mathrm{Sn}}-E_{\mathrm{In}}^{K}$ is $267 \mathrm{keV} .{ }^{3}$

Then the appropriate acceleration of ${ }_{50}^{110} \mathrm{Sn}$ is

$$
\gamma_{\mathrm{Sn}}=378\left(\frac{\delta m^{2}}{2.5 \times 10^{-3} \mathrm{eV}^{2}}\right)\left(\frac{L}{100 \mathrm{~km}}\right)\left(\frac{\pi / 2}{P}\right) .
$$

In the rest frame of ${ }_{50}^{110} \mathrm{Sn}$, the distance $L_{\mathrm{Sn}}^{\prime}$ is given by

$$
L_{\mathrm{Sn}}^{\prime}=264 \mathrm{~m}\left(\frac{2.5 \times 10^{-3} \mathrm{eV}^{2}}{\delta m^{2}}\right)\left(\frac{P}{\pi / 2}\right) .
$$

Therefore if the "fiducial" detector radius is larger than $264\left(\frac{P}{\pi / 2}\right) \mathrm{m}$, half the neutrinos goes into the detector. Because of the reason mentioned below the theoretically most interesting oscillation phase is $P=\pi / 3$ and hence $264\left(\frac{P}{\pi / 2}\right)=176 \mathrm{~m}$. This size of a detector is not unrealistic. Incidentally, since $\gamma_{\mathrm{Sn}}=567, \gamma_{\mathrm{Sn}} \tau_{\mathrm{Sn}}=96$ days, satisfying eq. (5). This efficiency should be compared with the case of a neutrino factory or a beta beam. In a neutrino factory [7] the distance, $L_{\mu}^{\prime}$ corresponding to $L_{\mathrm{Sn}}^{\prime}$ is $\mathrm{O}(10) \mathrm{km}$ and hence even if the area of the detector perpendicular to the neutrino beam is of $(\mathrm{O}(100) m)^{2}$, only $0.01 \%$ of the neutrinos are used. Similarly in a beta beam experiment $L_{\beta}^{\prime}$ is $O(1) \mathrm{km}$ and only $1 \%$ of neutrinos are used. Therefore, even if we have by 2 orders of magnitude a smaller amount of ${ }_{50}^{110} \mathrm{Sn}$ nuclei in decay than nuclei in a beta beam experiment, say ${ }_{2}^{6} \mathrm{He}$, we will have same reach for the physics. That is, the "quality factor" 8] is much better. Indeed $L^{\prime}$ is essentially the inverse of the quality factor. Furthermore, since the neutrino energy is much more clearly determined in this experiment, we have better precision.

There is another interesting feature in sufficiently high $\gamma_{m}$ experiment. As we have seen, almost all neutrinos go through the detector. Therefore we have a wide range of neutrino energies and from the detection point the neutrino energy is "measured" precisely. The energy of a neutrino, which is detected at $R$ away from the center of the beam, is easily calculated (in large $\gamma_{m}$ limit):

$$
E_{\nu}(R)=\frac{2 \gamma_{m} Q}{1+R^{2} / L^{\prime 2}}
$$

\footnotetext{
${ }^{2}$ Our picture for $\mathrm{K}$ shell electron capture is that a neutral mother captures its K shell electron and bears a neutral daughter with one $\mathrm{K}$ shell hall and one electron in outer orbit. Therefore, exactly speaking, we need to take into account the binding energy of an electron in the outer orbit, $E_{o}$ which will fall into the $\mathrm{K}$ shell finally. This raise the neutrino energy by amount of $E_{o}$, though we will omit this here.

${ }^{3}$ Since an electron is captured not only from K shell but also other orbits, there are several lines depending on from which shell an electron is captured. It should be included to consider the detail.
} 
The neutrino energy range is determined by eq.(8) ,

$$
\frac{2 \gamma_{m} Q}{1+D^{2} / L^{\prime 2}}<E_{\nu}<2 \gamma_{m} Q
$$

where $D$ is the "fiducial" detector diameter. For example, if $D=L^{\prime}$, then the half of emitted neutrinos hits the detector and their energy range is $\gamma_{m} Q \leq E_{\nu} \leq 2 \gamma_{m} Q$. The range of the oscillation phase varies from $\pi / 3$ to $2 \pi / 3$, from which we can explore the oscillation shape around the oscillation maximum very precisely.

For the position resolution $\delta R\left(\delta R^{2}=2 R \delta R\right)$, the energy resolution is given by

$$
\left|\delta E_{\nu}\right|=\frac{2 \gamma_{m} Q \delta R^{2} / L^{\prime 2}}{\left(1+R^{2} / L^{\prime 2}\right)^{2}} \Rightarrow\left|\frac{\delta E_{\nu}}{E_{\nu}}\right|=\frac{\delta R^{2} / L^{\prime 2}}{\left(1+R^{2} / L^{\prime 2}\right)} .
$$

In the rest frame of the mother nucleus, monoenergetic neutrino is emitted isotropically. In a solid angle $d \Omega$, the number of neutrinos distribute uniformly. The solid angle $d \Omega=2 \pi \sin \theta d \theta$ corresponds to

$$
2 \pi \sin \theta d \theta=\frac{4 \pi}{\left(1+R^{2} / L^{\prime 2}\right)^{2}} \frac{d R^{2}}{L^{\prime 2}}
$$

and in terms of the neutrino energy

$$
d \Omega=2 \pi \sin \theta d \theta=\frac{2 \pi}{\gamma_{m} Q} d E_{\nu}
$$

Thus we have the neutrino beam uniformly distributed in its energy. As a detector can measure the energy itself, by combining energy informations from the detection position, we can determine the neutrino energy very precisely. This specific feature in a beta-capture beam arises from the fact that neutrinos are monoenergetic in the rest frame of the mother nucleus.

In table. 【 we list nucleus candidates for this case (i).

case (ii) Monoenergetic neutrino and Continuous energy neutrino:

Next we consider the nuclei ${ }_{24}^{48} \mathrm{Cr}$. It decays into an excited state of ${ }_{23}^{48} \mathrm{~V}$ whose energy level is $420 \mathrm{keV}$. The mass difference is $1659 \mathrm{keV}$ and $\Delta_{\mathrm{Cr}}$ is $1239 \mathrm{MeV}$. The half-life is 21.56 hours. [10] $\mathrm{K}$ shell binding energy, $E_{\mathrm{V}}^{K}$, of the daughter nucleus ${ }_{23}^{48} \mathrm{~V}$ is $5.465 \mathrm{keV}[11]$. Since $Q_{\mathrm{Cr}}=\Delta_{\mathrm{Cr}}-E_{\mathrm{V}}^{K}$ is larger than $2 m_{e}$, twice of the electron mass, it not only captures an electron but also emits a positron:

$$
{ }_{24}^{48} \mathrm{Cr}+e^{-} \rightarrow{ }_{23}^{48} \mathrm{~V}+\nu_{e} \&{ }_{24}^{48} \mathrm{Cr} \rightarrow{ }_{23}^{48} \mathrm{~V}+e^{+}+\nu_{e}
$$




\begin{tabular}{|c|c|c|c|c|c|c|}
\hline Mother, $E^{K}[11]$ & Daughter, $E^{K}[11]$ & $\Delta[10]$ & $\tau[10]$ & $\gamma_{m}$ & $\tau \gamma_{m}$ & Detector Size \\
\hline \hline \begin{tabular}{c}
${ }_{50}^{10} \mathrm{Sn}, 29$ \\
\hline $\begin{array}{c}111 \\
49\end{array}$
\end{tabular} & $\begin{array}{c}{ }_{49}^{10} \mathrm{In}^{*}[343], 28 \\
{ }_{48} \mathrm{Cd}^{*}[417], 27\end{array}$ & 295 & $4.11 \mathrm{~h}$ & 567 & $97 \mathrm{~d}$ & $176 \mathrm{~m}$ \\
\hline
\end{tabular}

TABLE I: Nucleus candidates for case (i). $\gamma_{m}$ is determined by $P=\pi / 3$ for a detector at $L=$ $100 \mathrm{~km}$ and $\delta m^{2}=2.5 \times 10^{-3} \mathrm{eV}^{2}$ using eq.(4). The energy unit is keV. $\mathrm{N}^{*}[\mathrm{E}]$ means the excited state of the nucleus $\mathrm{N}$ with energy E[keV]. "Detector Size" indicates the radius within which a half of the emitted neutrinos are included at the detector distance, see eq.(2).

\begin{tabular}{|c|c|c|c|c|c|cc|}
\hline Mother, $\left.E^{K}[1]\right]$ & Daughter, $E^{K}[11]$ & $\Delta[10]$ & $\tau[10]$ & $\gamma_{m}$ & $\tau \gamma_{m}$ & EC : $e^{+}$emission \\
\hline \hline${ }_{9}^{18} \mathrm{~F}, 0.7$ & ${ }_{8}^{18} \mathrm{O}, 0.5$ & 1656 & $110 \mathrm{~m}$ & 123 & $4.65 \mathrm{~d}$ & $3.4:$ & 96.6 \\
\hline${ }_{24}^{48} \mathrm{Cr}, 6$ & ${ }_{23}^{48} \mathrm{~V}^{*}[420], 5$ & 1239 & $21.56 \mathrm{~h}$ & 82 & $74 \mathrm{~d}$ & $98.0:$ & 2.0 \\
\hline${ }_{50}^{111} \mathrm{Sn}, 29$ & ${ }_{49}^{111} \mathrm{In}, 28$ & 2445 & $35.3 \mathrm{~m}$ & 42 & $24.7 \mathrm{~h}$ & $40.5:$ & 59.5 \\
\hline${ }_{50}^{113} \mathrm{Sn}^{*}[77], 29$ & ${ }_{49}^{113} \mathrm{In}, 28$ & 1113 & $21.4 \mathrm{~m}$ & 93 & $33.2 \mathrm{~h}$ & $100:$ & 0 \\
\hline
\end{tabular}

TABLE II: Candidate Nuclei for case (ii). $\gamma_{m}$ is determined by $P=\pi / 2$ instead $\pi / 3$. The last column, the ratio of the electron capture and the positron emission, is calculated by using eq.(14) and eq.(15).

Assuming that there are $2 \mathrm{~K}$ shell electrons in the mother nucleus ${ }_{24}^{48} \mathrm{Cr}$, the rate for the capture process, $\Gamma_{c}$, is proportional to 12$]$

$$
\Gamma_{c} \propto 2 \pi\left\{\left(Q_{\mathrm{Cr}}\right) / m_{e}\right\}^{2}(\alpha Z)^{3}=0.196
$$

Here $m_{e}$ is the electron mass. The rate for positron emission , $\Gamma_{e^{+}}$, is proportional to [12]

$$
\begin{aligned}
& \Gamma_{e^{+}} \propto \int_{1}^{w_{0}} x \sqrt{x^{2}-1}\left(w_{0}-x\right)^{2} F(x, Z) d x=0.004 \\
& F(x, Z)=2(1+\gamma)\{2 p r\}^{2 \gamma-2} \exp (-\pi \nu) \frac{|\Gamma(\gamma-i \nu)|^{2}}{[\Gamma(2 \gamma+1)]^{2}}
\end{aligned}
$$

Here $F(x, Z)$ is the Fermi function $\left(\gamma \equiv(1-\alpha Z)^{1 / 2}, \nu \equiv \alpha Z x / p, p=\sqrt{x^{2}-1}, \alpha\right.$ the fine structure constant $=1 / 137$ and $r$ the radius for a nucleus in units of $\left.m_{e}^{-1}\right)^{4}$ and $w_{0}=$ $\left(\Delta_{\mathrm{Cr}}-m_{e}\right) / m_{e}$ is the maximum positron energy scaled by an electron mass. Thus the electron

\footnotetext{
${ }^{4}$ For numerical calculation we take $r=10^{-3}$. However the numerical results here does not depend on $r$ within a few $\%$ accuracy.
} 
capture process is dominant (98.0\%) and hence a neutrino beam with well-controlled energy is available.

In table we list other examples of nuclei which have still lower $Q$ and shorter $\tau[10] .{ }_{9}^{18} \mathrm{~F}$ dominantly decays by positron emission while ${ }_{24}^{48} \mathrm{Cr}$ and ${ }_{50}^{113} \mathrm{Sn}^{*}$ almost capture an electron to bear their daughter nucleus.

Since $Q_{\mathrm{Cr}}$ is higher than the previous case, the appropriate $\gamma_{\mathrm{Cr}}$ is lower and hence the quality factor is worse than the previous case. Namely, we need to prepare much more ${ }_{24}^{48} \mathrm{Cr}$ nuclei than ${ }_{50}^{110} \mathrm{Sn}$ :

$$
\gamma_{\mathrm{Cr}}=82\left(\frac{\delta m^{2}}{2.50 \times 10^{-3} \mathrm{eV}^{2}}\right)\left(\frac{L}{100 \mathrm{~km}}\right)\left(\frac{\pi / 2}{P}\right)
$$

which means that the neutrino at the detector is completely monoenergetic. There is essentially no position dependence of neutrino energy at the detector.

We also cannot explore the energy dependence of the oscillation simultaneously as previously discussed. However, this problem may be solved by the use of continuous neutrino associated with positron emission. We can control the boost factor $\gamma_{m}$ very well and hence the highest neutrino energy at a detector is completely determined from it. This offers very accurate calibration for neutrino energy. Furthermore, the energy of the line spectrum and that of the continuous one are clearly separated and simultaneous observation of two distinct energy region gives a useful information on Unitarity triangle 13]. Thus having a line and a continuous spectrum simultaneously, we may get better oscillation parameter reach.

We study how to control neutrino energy in oscillation experiments better than currently discussed ideas. By electron capture, a nucleus emits a monoenergetic neutrino. Therefore by accelerating the mother nuclei, we can get a well-controlled neutrino beam. To achieve $100 \%$ electron capture rate, we need to use a nucleus with a low $Q$ value, lower than $2 m_{e}$. In general, such a nucleus has a long half-life. Furthermore, since we accelerate it with significantly large boost factor $\gamma_{m}$, it becomes almost stable. Though this easily conflicts with the fact that the nucleus must decay within sufficiently short interval (see eq.(5) ), there are several candidates listed in table II. With these nuclei, we can control neutrino energy. Since $\gamma_{m}$ is very large, a neutrino beam is so well concentrated in the forward direction that almost all neutrinos can be used for oscillation experiments. It reduces significantly the necessary number of the mother nuclei. As a result of such a high $\gamma_{m}$, in principle, we don't 
need to measure the neutrino energy at a detector since by measuring the detected position we can calculate its energy and hence simultaneously we can observe the energy dependence of the oscillation.

Theoretically there are only advantages but these nuclei are so heavy that it is very energy consuming to accelerate them to an ideal $\gamma_{m}$. Also it may be hard to get enough nuclei even if the required number of nuclei is significantly small. As a compromise, we also study nuclei with a higher $Q$ value. Those nuclei not only capture an electron but also emit a positron. From the latter process neutrinos with continuous spectrum are emitted. Furthermore as $Q$ is higher, $\gamma_{m}$ must be smaller. These facts spoil some of the good features mentioned above. However since we have neutrinos with a line spectrum and a continuous spectrum simultaneously, we may get another good feature for this kind of beams.

In this kind of a beta-capture beam, we can produce only $\nu_{e}$ beam. To study CP violation we need $\bar{\nu}_{e}$ 14] or $\nu_{\mu}$ [15] beam. On the contrary to $e^{-}$capture case, since $e^{+}$cannot be bound by a nuclei, it is almost impossible to have a sufficiently strong $\bar{\nu}_{e}$ beam. Instead we can make use of $\mu$ capture to get monoenergetic $\nu_{\mu}$ beam, though since the mass of $\mu$ is very high, emitted neutrinos have a high energy. We must find a nucleus whose daughter has a mass higher than that of the mother by $\mathrm{O}(\mu)$ mass so that the energy of $\nu_{\mu}$ in the rest frame of the mother nucleus is sufficiently low.

Apart from the idea to make use of $e^{-}$capture, a nucleus ${ }_{9}^{18} \mathrm{~F}$ should be considered as the $\beta$ beam source more seriously. Note that in an ideal circumstance, with static strong magnetic large circulating ring, etc., we do not need any power supply to maintain current by nuclei. Therefore the mother nuclei do not have to decay "immediately" Since $\Delta_{\mathrm{F}}$ is $1655.5 \mathrm{keV}$ while $\Delta_{\mathrm{Ne}}$ is $4446 \mathrm{keV}$, we have much better "quality factor" than ${ }_{10}^{18} \mathrm{Ne} .{ }_{9}^{18} \mathrm{~F}$ is used for medical check, Positron Emission Tomography (PET). They are made within one hour about $\mathrm{O}\left(10^{10}\right) \mathrm{Bq}$, about $10^{14}$ nuclei per hour $\simeq 10^{18}$ nuclei per year even in a medical check. We can use a much larger amount of such a nuclei much more easily than ${ }_{10}^{18} \mathrm{Ne}$. Similarly we need to reconsider a candidate nuclei for $\bar{\nu}_{e}$ source with lower $Q$ than ${ }_{2}^{6} \mathrm{He}$, e.g. ${ }_{14}^{31} \mathrm{Si}$.

\section{Acknowledgments}

The author thanks T. Ota for useful comment. The author also thanks T. Higuchi and M. Ishiduki about information on PET. He is supported by the Grant-in-Aid for Scientific 
Research on Priority Area No.16038202 and 14740168.

[1] B. T. Cleveland et al., Astrophys. J. 496 (1998) 505; SAGE Collaboration, J. N. Abdurashitov et al., J. Exp. Theor. Phys. 95 (2002) 181 ; GALLEX Collaboration, W. Hampel et al., Phys. Lett. B447 (1999) 127; GNO Collaboration, M. Altmann et al., Phys. Lett. B490 (2000) 16.; Super-Kamiokande Collaboration, M. Smy et al., Phys. Rev. D69 (2004) 011104; SNO Collaboration, S. N. Ahmed et al., Phys. Rev. Lett. 92 (2004) 181301;

[2] Super-Kamiokande Collaboration, Y. Ashie et al., arXiv:hep-ex/0501064; Phys. Rev. Lett. 93 (2004) 101801; G. Giacomelli and A. Margiotta, Phys. Atom. Nucl. 67 (2004) 1139 ; Soudan 2 Collaboration, M. Sanchez et al., Phys. Rev. D68 (2003) 113004.

[3] KamLAND Collaboration, T. Araki et al., arXiv:hep-ex/0406035 K. Eguchi et al., Phys. Rev. Lett. 90 (2003) 021802.

[4] K2K Collaboration, M. H. Ahn et al., Phys. Rev. Lett. 90 (2003) 041801.

[5] e.g. M.C. Gonzalez-Garcia and C. Pena-Garay, Phys. Rev. D68 (2003) 093003.

[6] JHF-Kamioka project, Y. Itow et al., arXiv:hep-ex/0106019.

[7] Neutrino Factory and Muon Collider Collaboration, C. Albright et al., arXiv:physics/0411123; NuFACTJ, http://psux1.kek.jp/nufact01/report.html.

[8] P. Zucchelli, Phys. Lett. B (2002) 166 .

[9] M. Mezzetto, arXiv:hep-ph/0410083.

[10] R. B. Firestone and V. S. Shirley, Table of Isotopes, 8th ed. (John Wiley \& Sons Inc 1996).

[11] J. A. Bearden and A. F. Burr, Rev. Mod. Phys. 39, (1967) 125.

[12] M. Fukugita and T. Yanagida, "Physics of Neutrinos and applications to Astrophysics", (Springer-Verlag, Germany, 2003).

[13] J. Sato, Nucl. Instrum. Meth. A472 (2000) 434.

[14] J. Arafune and J. Sato, Phys. Rev. D55 (1997) 1653.

[15] J. Arafune, M. Koike and J. Sato , Phys. Rev. D56 (1997) 3093; Erratum-ibid. D60 (1999) 119905. 